\title{
Rol parental educativo: aproximación a las percepciones que poseen los futuros profesores ${ }^{1}$
}

Héctor Cárcamo-Vásquez

Universidad del Bío Bío (Chile)

hcarcamo@ubiobio.cl
Carlos Rodríguez-Garcés

Universidad del Bío Bío (Chile)

carlosro@ubiobio.cl

\section{Resumen}

El artículo tiene por objetivo reconocer las percepciones que poseen los alumnos que cursan su formación inicial docente respecto de las familias y del modo en que estas se relacionan con la escuela. La metodología utilizada fue cuantitativa y el tipo de estudio de alcance descriptivo. La información se obtuvo a través de un cuestionario autoaplicado que contempla la escala de percepción docente sobre participación de padres y madres en la escuela, elaborado por Martín y Armas (2011). Los hallazgos más relevantes permiten sostener que los profesores en formación vislumbran el rol parental educativo como relevante y complementario a la acción pedagógica para el logro educativo, siempre que se exprese fuera de la escuela y no vinculado directamente con el quehacer docente. Imagen que es complementada con la percepción negativa y deteriorada que se tiene respecto de las familias de los educandos en su relación con el proceso educativo de sus hijos.

\section{Palabras clave}

Contextos educativos, informática educativa, métodos de enseñanza, formación de profesores en servicio (Fuente: Tesauro de la Unesco).

1 Artículo derivado del proyecto de investigación "La relación familia-escuela como espacio de construcción de la ciudadanía en espacios educativos formales", código: DIUBB 142424 2/RS, financiado por la Dirección de Investigación de la Universidad del Bío-Bío, Chile y el proyecto CD 12O2-4, financiado por el Convenio de Desempeño Sistema Territorial de Educación (MECESUP-UBB CD12O2).

Recepción: 16-01-2015 | Envío a pares: 09-02-2015 | Aceptación por pares:22-05-2015 | Aprobación: 24-05-2015 DOI: 10.5294/edu.2015.18.3.5

Para citar este artículo / To reference this article / Para citar este artigo

Cárcamo-Vásquez, H., Rodríguez-Garcés, C. (2015). Rol parental educativo: aproximación a las percepciones que poseen los futuros profesores. Educ. Educ., 18 (3), 456-470. DOI: 10.5294/edu.2015.18.3.5 


\title{
Parental Role in Education: Approach to the Perceptions Held by Future Teachers
}

\begin{abstract}
The purpose of this study is to identify what students in initial teacher training think about families and their relationship to the school. A quantitative method was used and the research was descriptive. A self-administered questionnaire was applied to collect the data. It included the scale of teacher's perception of parental involvement in school developed by Martin and Armas (2011). The most relevant findings indicate teacher trainees see the parental role in education as relevant and complementary to teaching, provided it is expressed outside the school and not linked directly to the work of teaching. This image is accompanied by the negative and deteriorated perception teacher trainees have of parental involvement in children's educational process.
\end{abstract}

\section{Keywords}

Educational contexts, computer education, teaching methods, in-service teacher training (Source: Unesco Thesaurus). 


\section{Papel parental educativo: uma aproximação às percepções que os futuros professores possuem}

\section{Resumo}

O artigo tem por objetivo reconhecer as percepções que os alunos que cursam sua formação inicial docente possuem com relação às famílias e ao modo como estas se relacionam com a escola. A metodologia utilizada foi quantitativa e o tipo de estudo de alcance descritivo. A informação obteve-se por meio de um questionário autoaplicado que contempla a escala de percepção docente sobre a participação de pais e mães na escola, elaborado por Martín e Armas (2011). As descobertas mais relevantes mostram que os professores em formação vislumbram o papel parental educativo como relevante e complementário à ação pedagógica para o sucesso educativo, sempre que se expresse fora da escola e não vinculado diretamente com o papel docente. Imagem que é complementada com a percepção negativa e deteriorada que se tem com relação às famílias dos educandos em sua relação com o processo educativo de seus filhos.

\section{Palavras-chave}

contextos educativos, informática educativa, métodos de ensino, formação de professores em serviço (Fonte: Tesauro da Unesco). 


\section{Introducción}

No cabe duda de la importancia que se ha otorgado al papel desempeñado por la familia y por la escuela en los procesos de socialización, estableciéndose tempranamente una diferenciación entre ambas instituciones de acuerdo con el peso que se considera que cada una de ellas posee según la edad o etapa vital en la que se encuentre el niño. Diversos autores, entre los que destacan Bas y Pérez (2010), Cerletti (2010), Feito (2010), Garreta y Llevot (2011), Gubbins (2012), dan cuenta de la necesidad de articular el análisis desde una lógica de continuidad entre los agentes, evidenciando la necesidad de configurar una relación familia-escuela dialógica, fluida, estable y comprometida.

En Chile, la discusión en torno a la relación familia escuela en el contexto de las reformas educativas es más bien marginal (Bellei, Muñoz, Pérez y Raczinsky, 2003; Cox, 2003; Donoso, 2004); no obstante, se reconoce que una estrecha relación entre las familias y el profesorado reviste evidentes beneficios para los alumnos, tal como lo sostienen autores como Sampé, Arandia y Elboj (2012), Aubert, Elboj, García y García (2010), Bellei, Gubbins y López (2002), Epstein (1995), Fernández (2007), Gallardo (2006), Londoño y Ramírez (2012), Walker y Hoover-Dempsey (2008), entre otros. En este sentido, las reformas educativas desarrolladas en Chile, en especial a partir de 1990, han hecho eco de esta situación, asumiendo la responsabilidad de diseñar e implementar un marco normativo que regule de forma adecuada la relación que se establece entre las familias y el profesorado. A este respecto, cabe señalar que uno de los pilares de la reforma de la educación desarrollada en los años noventa es el eje de participación de padres y apoderados en la educación (Ministerio de Educación de Chile, 2004). Precisamente, desde el Ministerio de Educación de Chile, tal como nos lo indican Bellei, Gubbins y López (2002), se propone una progresiva descentralización administrativa y financiera, propiciando que en cada escuela se considere la realidad particular de su zona y la incorpo- ración activa de la comunidad donde esté inserta, favoreciendo de esta forma la distribución innovadora del poder al interior de las escuelas. A modo de ilustración, podemos señalar que las familias pueden participar en la elaboración del Proyecto Educativo Institucional (PEI) de sus escuelas, así como también de la creación del Reglamento de Convivencia y los Consejos Escolares.

Ahora bien, la normativa es letra muerta si no cuenta con la convicción y la adhesión del profesorado. De ahí la importancia de estudiar las percepciones que los alumnos que cursan su formación inicial docente tienen respecto a esta temática. Pues, de acuerdo con Garreta (2009), las expectativas que las familias depositan en la educación intervienen directamente en el tipo de relación que establecen con la escuela. No obstante, el grado de compromiso e implicación de las familias no depende exclusivamente del interés que estas tengan en comprometerse e implicarse, sino también de la actitud que el profesorado adopte con respecto a dicho compromiso e implicación (Moscoso, 2009; Pozo, 1996). En este sentido, Walker y Hoover-Dempsey (2008) plantean que el profesorado que posee una actitud positiva respecto a la participación de las familias en las escuelas obtiene importantes beneficios, pues logra conocer a las familias de sus alumnos, facilitando no solo la participación sino también el desarrollo más fluido y estrecho de la relación. En consecuencia, las percepciones que los futuros profesores tengan de las familias y del modo en que estas se relacionan con la escuela, son relevantes para entender cómo se van a vincular en el futuro con ellas en su ejercicio profesional. Tal como afirman Garreta y Llevot (2011), Gubbins (2012), Olmsted (1991), Pérez, Cortese y Gallardo (2007), Roldán (2008), Santín (2006), el tipo de relación que se establece entre las familia y la escuela está mediada, en términos generales, por las teorías implícitas o explícitas que los profesores tienen respecto de lo que hemos denominado rol parental educativo. Por otra parte, autores como Cárcamo (2013), Feito (2010), Garreta (2008), Pascual y Gomila 
(2012), Santos Guerra (2010) sostienen que para asegurar la participación de las familias y una buena relación con ellas, hay que comenzar interviniendo en la formación inicial del profesorado. Intervención relevante toda vez que, quienes estudian para ser profesor, suelen tener una imagen negativa de la familia y en particular de su rol parental educativo.

Investigaciones recientes (Cárcamo, 2012; Pascual y Gomila, 2012; Poveda, Jociles y Franzé, 2014; Rio, 2010) han constatado que los futuros maestros de primaria tienden a externalizar, o situar fuera de la escuela la responsabilidad del bajo rendimiento académico de los alumnos. Responsabilizan a las familias cuando este rendimiento es bajo. Mientras, el alto rendimiento es atribuido al buen hacer del profesorado o a la buena gestión de los centros escolares. De igual modo, siguiendo los planteamientos de Río (2010), el discurso de los profesores oscilaría entre dos juicios. Por una parte, el que mantiene que las familias no saben y no pueden intervenir de manera adecuada en la educación escolar de sus hijos; y, por otra, el que les recrimina que no quieren saber hacerlo. Ambos juicios aluden a la falta de capacidad o voluntad de las familias para ajustarse a la norma escolar, máxime cuando de familias en riesgo psicosocial o con altos niveles de vulnerabilidad se trata. En estas nociones priman dos criterios de clasificación. Por un lado, tenemos la noción de funcionalidad/disfuncionalidad familiar enraizada en el discurso sociológico; y por otro, la noción de déficit cultural de las familias y su distancia normativa respecto de la escuela, idea proveniente de la antropología de la educación.

La externalización de responsabilidades se apoya en la representación de un ideal de padres y en el compromiso como eje principal de significación (Jociles, Franzé y Poveda, 2012). Los padres y apoderados ideales son quienes se comprometen con la educación de sus hijos, asistiendo a las entrevistas individuales concertadas por el profesor y a las reuniones convocadas por este, además de apoyar a sus hijos en la realización de tareas y promover adecua- dos hábitos de estudio en casa (Armas, 2012; Oraisón y Pérez, 2006; Pascual y Gomila, 2012; Pérez et al., 2007). Aspectos estos últimos que, si bien no pueden ser observados directamente por el profesor, son cotejados mediante la revisión de los deberes que cada niño lleva al colegio. En suma, el profesorado espera que el rol que cumplan los padres con respecto a la escuela se restrinja a los ámbitos predefinidos desde ella (Garreta y Llevot, 2011). En consecuencia, la participación parental educativa se hace en un espacio especialmente creado por la escuela para ser llenado por las familias (Cornwall, 2002; Gaventa, 2005); espacio que es restringido en atención a la desconfianza que evidencia el profesorado respecto de la participación de los padres por considerarlos no capacitados para la tarea de enseñar. Idea que es modelada a partir de una profesionalidad clásica en la que subyace un distanciamiento entre maestros y padres que impide la colaboración entre los agentes (Bolívar, 2006; Núñez, Arévalo y Ávalos, 2012; Pascual, 2002; Torres, 1999-2000; Vera, 1987).

Sobre la base de los elementos encontrados en la literatura, este trabajo se propone dar cuenta de la percepción que posee el profesorado en formación respecto del cumplimiento del rol parental educativo que asignan a las familias. En particular, se caracterizan las percepciones que posee el profesorado en formación respecto de las familias, el modo en que estas se relacionan con la escuela y los roles que consideran deben cumplir. Configurando el nivel de participación deseable que los profesores esperan alcancen las familias en el contexto escolar para dar cumplimiento al rol parental educativo, entendido como el conjunto de acciones y tareas que según el profesorado debe llevar a cabo la familia para apoyar la educación formal de sus hijos.

\section{Materiales y métodos}

Para el desarrollo de esta investigación utilizamos la metodología cuantitativa de alcance descriptivo. La población de estudio estuvo compuesta por 
los alumnos que cursan su formación inicial docente en la carrera de Pedagogía en Educación Básica en una universidad pública regional del sur de Chile.

En cuanto a la técnica para el levantamiento de información, se utilizó una encuesta autoaplicada durante el periodo lectivo del año 2014. Este cuestionario ad hoc contempla la escala de percepción docente sobre participación de padres y madres en la escuela elaborado por Martín y Armas (en Armas, 2012), que consta de 37 ítems tipo likert de respuesta graduada en 5 niveles, los cuales transitan desde nada de acuerdo a totalmente de acuerdo. El análisis de validación precedente arroja un índice de confiabilidad alfa de Cronbach de o,8. Índice que asegura una apropiada consistencia interna.

El instrumento se aplica a 175 alumnos de un total 259 que cursan su formación inicial docente, los cuales corresponden al $68 \%$ de la población. El muestreo fue aleatorio estratificado buscando la representación proporcional según cohorte de ingreso. Tamaño muestral y procedimiento que aseguran una adecuada representación.

El contingente encuestado (tabla 1) corresponde en un 67,4\% a la cohorte de ingreso 2012-2014, por lo que 4 de cada 10 alumnos consultados tienen una experiencia de formación inicial docente de al menos 4 años. La edad promedio de los encuestados es 22,5 años, donde un 53,1\% registra menos de 22 años. La distribución por sexo, siguiendo tendencias nacionales históricas, muestra una clara supremacía de las mujeres (80\%). En lo relativo al establecimiento de procedencia, podemos señalar que la mayoría de los sujetos provienen de establecimientos particulares subvencionados $(67,6 \%)$, seguidos de colegios municipalizados (31,8\%), de modo que la presencia de alumnos provenientes de colegios particulares pagados es marginal (0,6\%), hecho que encuentra su explicación en procesos de segmentación socioeducativa que caracterizan el sistema escolar chileno y se prolongan en el espacio universitario, haciendo que la elección profesional no solo se vincule con aspectos meramente vocacionales.
Tabla 1.

\section{Caracterización de la muestra (porcentajes)}

\begin{tabular}{|l|c|}
\hline & $\%$ \\
\hline Edad: & \\
\hline - 18 a 21 & 53,1 \\
- 22 a 26 & 34,4 \\
- > 26 & 12,5 \\
\hline Sexo: & \\
\hline - Hombre & 20,0 \\
- Mujer & 80,0 \\
\hline Establecimiento de procedencia: & \\
\hline - Municipal & 31,8 \\
- Particular subvencionado & 67,6 \\
- Particular pagado & 0,6 \\
\hline Cohorte de ingreso: & \\
\hline - 2005-2011 & 32,6 \\
- 2012-2014 & 67,4 \\
\hline Vive con: & \\
\hline - Ambos padres & 68,0 \\
- Solo con madre/padre & 24,5 \\
- Emancipado & 7,4 \\
\hline Nivel educativo madre: & \\
\hline - o a 8 k & 36,0 \\
- 11 a 12 k & 59,4 \\
- > de 12 k & 4,6 \\
\hline Nivel educativo padre: & \\
\hline - o a 8 k & 36,2 \\
- 11 a 12 k & 55,2 \\
- > de 12 k & 8,6 \\
\hline
\end{tabular}

En cuanto a la estructura familiar de los alumnos, cabe indicar que se ajusta a lo convencional, apreciándose una clara presencia de familias nucleares, pues un 68,0\% vive con ambos padres, seguido de familia monoparental con un $24,5 \%$, con clara predominancia de jefatura femenina. Asociado a elementos de ciclo vital, así como a dependencia económica, un escaso $7,4 \%$ vive sin sus padres. Finalmente, de acuerdo con el nivel educativo parental, dos tercios de los encuestados corresponden a la primera generación que asiste a la universidad, por cuanto solo un 4,6\% de las madres alcanza un nivel de escolaridad superior a la enseñanza media completa (12 k), y el 8,6\% de los padres. 
Para efecto de análisis, y en razón de que se buscaba centrar la atención en las percepciones que los futuros docentes tenían respecto del conjunto de acciones y tareas que deben llevar a cabo las familias para apoyar la educación formal de sus hijos, se exploraron las siguientes variables que conforman el rol parental educativo:

Tipos y niveles de participación expresados como informativo, propositivo y consultivo.

Beneficios atribuidos a la relación familia-escuela, que en un conjunto de 9 ítems de respuesta graduada explora el nivel de acuerdo respecto de los efectos asignados por el docente a la implicación parental.

Percepción parental: analiza, mediante 8 items, las concepciones y significaciones que tienen y hacen los profesores de las familias en relación con el proceso educativo de sus hijos, el nivel de compromiso y sintonía que exhiben con el quehacer del profesor.

Cumplimiento del rol parental educativo: analiza, mediante 6 items, el nivel de adhesión que los padres exhiben en la actualidad respecto de las funciones que a juicio del profesor constituyen un adecuado rol educativo e implicación.

Los datos fueron procesados mediante el uso del software SPSS en su versión 21.0, específicamente los módulos de contraste de medias (prueba $t$ de student para muestras relacionadas), tablas de contingencia y contraste de proporciones.

\section{Resultados}

Respecto a la percepción que manifiestan los profesores en formación inicial en torno a la participación y el rol que deberían jugar las familias en el proceso educativo de sus hijos, en especial en la relación familia-escuela (tabla 2), se constata una mayor sintonía con el nivel de participación parental educativa de tipo informativo ( $\bar{x}: 4,35+/-0,88)$, que representaría, a juicio de la literatura consultada, el nivel más básico de participación. Este componente dista significativamente de los niveles consultivo [ $(\overline{\mathrm{x}}$ : 3,60 +/- 1,07); (tım: -9,3; gl: 174; a < 0,01)] y propositivo [( $\left.\bar{x}: 3,64+/-1,16) ;\left(t_{1 m}:-6,9 ; g l: 173 ; a<0,01\right)\right]$. Hallazgos que son consistentes con los obtenidos en investigaciones que exploran el nivel de participación alcanzado por las familias en los centros escolares por Armas (2012), Garreta y Llevot (2011), Gubbins (2012), Ramírez (2008), Walker y Hoover-Dempsey (2008), entre otros. En efecto, en lo que a nuestros datos respecta, y en concordancia con los niveles de participación analizados, los sujetos señalan que "la información es la clave de la participación ya que aumenta el interés de los padres" (83,2\% muy de acuerdo y totalmente de acuerdo), que "informar a los padres sobre las decisiones que se toman en el establecimiento es una forma de participación" (78,2\% muy de acuerdo y totalmente de acuerdo). En contraposición, en reactivos que ponen el acento en tipologías de participación distintas de la informativa, se constata un nivel de aceptación significativamente más reducido. Así por ejemplo, respecto de "solicitar opinión y participación en la formulación de propuestas a los padres, aunque la decisión final la toma el establecimiento", tan solo un $56,6 \%$ se manifiesta muy de acuerdo y totalmente de acuerdo, índice que reduce aún más respecto de que "los padres deben tomar parte en la formulación, análisis y evaluación de propuestas del establecimiento" (41\% muy de acuerdo y totalmente de acuerdo).

Tabla 2. Nivel de participación que consideran deben cumplir las familias en el marco del rol parental educativo (medias)

\begin{tabular}{|l|c|}
\hline Nivel de participación & $\overline{\mathrm{x}}(\mathrm{s})$ \\
\hline Informativo & $4,35(0,88)$ \\
\hline Consultivo & $3,60(1,07)^{*}$ \\
\hline Propositivo & $3,64(1,16)^{*}$ \\
\hline
\end{tabular}

* Informa diferencias estadísticamente significativas de la categoría respecto de nivel de participación informativa $\left(t_{1 m .} . a<0,01\right)$. 
Esta situación puede entenderse a partir de la noción de profesionalidad clásica, base desde la cual se modelan y definen las fronteras del desempeño de la profesión docente (Núñez et al., 2012; Vera, 1987). Estas fronteras demarcan los límites entre el saber hacer desde la escuela frente al apoyo solicitado a las familias para la consecución de objetivos previamente trazados. En consecuencia, el rol parental educativo es percibido por el profesor desde una perspectiva utilitaria y complementaria a su quehacer en la medida que este se ejerce en el espacio de lo doméstico, desde donde los padres colaboran en lo definido y estructurado por el profesor. Es desde este saber experto que la función familiar educativa se traduce en la instalación de hábitos de estudio, cumplimiento de tareas y quehaceres, incentivo de la lectura, así como la toma de medidas remediales de reforzamiento al déficit de aprendizaje o bajo nivel de logro detectado e informado por el profesor. Asimismo, cuando el rol parental educativo traspasa esta frontera de lo informativo buscando un mayor protagonismo en el proceso educativo de sus hijos, las familias son percibidas como agentes potencialmente disruptivos.

No obstante asignar a padres y apoderados una función complementaria con un nivel básico de par- ticipación de tipo informativo, los futuros profesores reconocen un conjunto de beneficios que reviste el establecimiento de una estrecha y fluida relación familia-escuela (tabla 3). Dentro de los atributos más destacados de esta relación encontramos la valoración que se hace del rol parental educativo en lo referente a la consecución del éxito académico del alumno. Se estima que "la implicación de los padres en la educación de sus hijos mejora su rendimiento académico" (86,2\%), y que una relación positiva familia-escuela "...eleva el rendimiento escolar de los alumnos" $(88,4 \%)$ y "...previene el fracaso escolar" (74,1\%). En consecuencia, las percepciones que configuran el perfil actitudinal de los sujetos se estructuran con base en los beneficios que otorga el rol parental educativo en el plano de lo académico y, por tanto, de impacto directo en el nivel de desempeño del profesorado como expresión del logro que alcancen los educandos. Estos elementos encuentran su correlato en el discurso académico proveniente de diversas disciplinas de las ciencias sociales que circulan sobre esta temática, pues parece haber una suerte de consenso en torno a la importancia que reviste el cumplimiento del rol parental educativo para asegurar el buen desempeño académico, tal como lo indican Garreta y Llevot (2011), Gubbins (2012), Sampé et al. (2012).

Tabla 3. Beneficios que reviste la relación familia-escuela (porcentajes y medias)

\begin{tabular}{|l|c|c|}
\hline \multicolumn{1}{|c|}{ Item } & $\%^{*}$ & $\overline{\mathrm{x}}(\mathrm{s})$ \\
\hline Para conocer las características de los alumnos es necesario contactar a los padres & 78,7 & $4,11(0,97)$ \\
\hline La participación de los padres es un factor decisivo para la calidad de la educación & 74,6 & $4,07(0,93)$ \\
\hline La implicación de los padres en la educación de sus hijos mejora su rendimiento académico & 86,2 & $4,39(0,77)$ \\
\hline Una relación positiva escuela-familia eleva el rendimiento escolar de los alumnos & 88,4 & $4,45(0,77)$ \\
\hline Una buena relación familia-escuela previene el fracaso escolar & 74,1 & $3,98(1,23)$ \\
\hline La participación de los padres en el establecimiento reduce los conflictos & 49,4 & $3,60(0,98)$ \\
\hline Oue los padres participen en el establecimiento genera problemas & 7,5 & $1,96(1,02)$ \\
\hline $\begin{array}{l}\text { Los padres que no están implicados en la educación de sus hijos tienen una actitud negativa } \\
\text { hacia la escuela }\end{array}$ & 33,9 & $3,13(1,11)$ \\
\hline La participación de los padres puede convertirse en una intrusión en el campo profesional & 19,8 & $2,81(0,93)$ \\
\hline
\end{tabular}

* Las cifras expresan porcentajes agrupando las categorías de respuesta Muy de acuerdo y Totalmente de acuerdo para cada uno de los ítems. 
Este reconocimiento a la contribución que pueden realizar los padres en el mejoramiento del desempeño académico de sus hijos no es tan solo complementario con la labor docente, sino que además dicha función no generaría tensiones en la medida en que se ejerce fuera del aula y de los tiempos lectivos. Cuando se traspasan dichas fronteras, la participación de los padres puede ser concebida como potencialmente amenazante. Consistente con dicha creencia, y si bien un reducido porcentaje considera que cuando "los padres participan en el establecimiento genera problemas" (7,5\%), tan solo un 33,3\% está a favor de "la implicación de los padres en actividades dentro del aula", y que con ella se corre el riesgo de "convertirse en una intrusión en el campo profesional" (19,8\% totalmente de acuerdo y muy de acuerdo). Concepciones que asocian a la participación como deseable y adecuada en la medida que se circunscribe a su nivel informativo, de rol pasivo y de accionar no amenazante.

La representación que tienen y hacen los profesores en formación respecto del rol parental educativo es vislumbrada como relevante y complementaria a la acción pedagógica para el logro educativo en cuanto se exprese fuera de la escuela y no vinculado directamente con el quehacer docente. Imagen que es complementada con la percepción negativa y desmejorada que se tiene respecto de las familias de los educandos en su relación con el proceso educativo de sus hijos (tabla 4). A juicio de los sujetos encuestados, los padres y apoderados no solo cuestionan el quehacer del profesorado (71,8\% totalmente de acuerdo y muy de acuerdo), sino que además delegan "la responsabilidad de educar a la escuela" (73,4\%), "usan la escuela como guardería" (66,7\%), se despreocupan de la formación en valores de sus hijos $(47,1 \%)$.

Si bien estos resultados son bastante coincidentes con hallazgos de otras investigaciones relativas a esta temática, dentro de las que se destacan la de Armas (2012), Cárcamo (2013) y Pascual y Gomila (2012), sorprende la magnitud o intensidad en que se expresa esta imagen deteriorada de la familia por parte del profesorado en formación. Esta representación desmejorada del cumplimiento del rol parental educativo tendría cierta base objetiva que la ampara. Las nuevas pautas de consumo familiar, mejores perspectivas laborales, así como una más positiva actitud frente al trabajo, conminan o facilitan a ambos padres ser perceptores de ingreso e insertarse en el mercado laboral, delegando en mayor medida el cuidado y la formación de los hijos a la escuela, evento que se suscita cada vez a más temprana edad. La irrupción de la mujer al mercado laboral, en especial cuando esta es madre y jefa de hogar, así como el aumento significativo de la cobertura de la educación preescolar son expresión de este fenómeno. Esto complejiza la labor docente y da cuenta de una realidad diferente a la de antaño, para lo cual los profesores no se sienten o están suficientemente preparados, al tener que conciliar la labor pedagógica de instalar competencias cognitivas con una preocupación por brindar condiciones de educabilidad.

\section{Tabla 4. Percepción respecto de las familias y su relación con el proceso educativo de sus hijos (porcentajes y medias)}

\begin{tabular}{|l|c|c|}
\hline \multicolumn{1}{|c|}{ Item } & $\%^{*}$ & $\overline{\mathrm{x}}(\mathrm{s})$ \\
\hline $\begin{array}{l}\text { Cuestionan el quehacer del } \\
\text { profesorado }\end{array}$ & 71,8 & $4,09(0,99)$ \\
\hline $\begin{array}{l}\text { Participan de reuniones } \\
\text { convocadas }\end{array}$ & 48,6 & $3,42(1,04)$ \\
\hline $\begin{array}{l}\text { Usan las escuela como } \\
\text { "guardería" }\end{array}$ & 66,7 & $3,88(1,09)$ \\
\hline $\begin{array}{l}\text { Se relacionan mínimamente con } \\
\text { los profesores }\end{array}$ & 44,8 & $3,43(0,96)$ \\
\hline $\begin{array}{l}\text { Se despreocupan de la } \\
\text { formación en valores de sus } \\
\text { hijos }\end{array}$ & 47,1 & $3,43(1,11)$ \\
\hline $\begin{array}{l}\text { Delegan responsabilidad a } \\
\text { escuela y profesores }\end{array}$ & 73,4 & $4,03(0,99)$ \\
\hline
\end{tabular}

* Las cifras expresan porcentajes agrupando las categorías de respuesta Muy de acuerdo y Totalmente de acuerdo para cada uno de los ítems. 
Las representaciones respecto del rol parental educativo que poseen los futuros profesores de educación básica no solo hacen mención a una familia despreocupada y escasamente diligente en el proceso formativo de sus hijos, sino que además esta imagen dista de manera significativa respecto de la atribuida a familias de antaño. Es en este sentido que, desde una perspectiva comparada (familias actuales, familias de compañeros y familia propia), se observan diferencias significativas respecto de la percepción del apoyo familiar en el proceso educativo. En ítems de gradiente ascendente de 1 a 10 en el apoyo familiar al proceso educativo de los hijos, los futuros profesores tienden a valorar más favo-


que la ajena, esté referida a la experiencia familiar de sus compañeros [( $\bar{x}: 6,63+/-1,79$; trel:-12,29; gl: 173; $\mathrm{a}<0,01)]$ o a las familias actuales [( $\bar{x}: 6,03+/-1,76$; trel: -13,37; gl: 173; a < 0,01)]. Si bien en las experiencias narrativas y evaluativas se tiende a apreciar una lógica de discontinuidad entre la experiencia propia y la de otros (Cárcamo, 2013), estas mismas diferencias se acentúan de forma evidente cuando se incorpora la distinción de carácter temporal comparando las familias de antes con las actuales. Distinción que encontraría su explicación, entre otras causas, en el discurso sociológico que permea la formación del profesorado respecto de la estructura y la función de las familias en escenarios cambiantes y frágiles (Bauman, 2002; Fernández, 2007; Garreta, 2008), llevando a idealizar la imagen de las familias de antaño, exagerando la sintonía que tendrían con el rol parental educativo, sobre todo cuando de la propia experiencia se trata, en detrimento de la visión que se tiene de las familias actuales.

No obstante la evocación idealizada del pasado que tiende a exagerar los atributos de las familias de antes, así como la percepción de pérdida de sintonía de las familias actuales con el quehacer docente con ocasión de la modernidad y las nuevas pautas de consumo, el profesorado en formación atribuye al rol parental educativo una función preponderan-
Tabla 5. Tareas consideradas relevantes en el cumplimiento del rol parental educativo (porcentaje)

\begin{tabular}{|l|c|}
\hline \multicolumn{1}{|c|}{$\begin{array}{c}\text { Tareas comprendidas para el rol parental } \\
\text { educativo }\end{array}$} & $\%^{*}$ \\
\hline Ayudar en las tareas escolares & 85,1 \\
\hline Promover hábitos de estudio & 86,8 \\
\hline $\begin{array}{l}\text { Asistir a reuniones de apoderados y } \\
\text { entrevistas con el profesor }\end{array}$ & 76,4 \\
\hline $\begin{array}{l}\text { Colaborar en actividades programadas por } \\
\text { el profesor }\end{array}$ & 21,3 \\
\hline $\begin{array}{l}\text { Participar en Centro General de } \\
\text { Padres y Apoderados y actividades del } \\
\text { establecimiento }\end{array}$ & 9,8 \\
\hline Incentivar la lectura & 75,9 \\
\hline
\end{tabular}

* Las cifras expresan porcentajes de respuestas emitidas en razón del formato de naturaleza de elección múltiple de la pregunta.

te en el proceso formativo de sus hijos, siempre y cuando este se materialice en el cumplimiento de tareas fuera del espacio escolar. En este sentido, se visualiza como relevante el "promover hábitos de estudio" (86,8\%), "ayudar en las tareas escolares" (85,1\%), "asistir a reuniones de apoderados y entrevistas con el profesor" (76,4\%) e "incentivar la lectura" (75,9\%). Conjunto de tareas que, exceptuando la asistencia a reuniones, tienen su espacio de desarrollo fuera del establecimiento educacional. Complemento del quehacer educativo que se viene a reflejar como base justificante de la externalización de responsabilidades en torno al fracaso escolar, tal como lo indican Jociles et al. (2012).

Al momento de comparar en qué medida estas actividades percibidas como definidoras del rol parental educativo formaron parte de la experiencia familiar del sujeto, se constatan diferencias de relevancia, observándose un menor apoyo familiar percibido en materia educativa. En contraste con la relevancia que los futuros profesores le asignan al cumplimiento de estas tareas, sus padres habrían otorgado una menor importancia a la instalación de hábitos de estudio e incentivo de la lectura, y habrían 
tenido una menor injerencia en el acompañamiento en las tareas escolares. Esta diferencia perceptiva no solo es expresión de la natural distancia existente a nivel evaluativo entre la experiencia vivida y la idealización del componente normativo, sino que también es expresión de los contextos socioculturales de sus trayectorias vitales. En efecto, el cambio intergeneracional, en especial el referente a capital humano acumulado, se profundiza cuando de profesores en formación se trata, quienes en su gran mayoría pertenecen a los quintiles de menores ingresos, son primera generación en cursar estudios superiores y pertenecen a los segmentos socioeducativos de mayor vulnerabilidad en la calidad de sus procesos formativos. En consecuencia, más que un desentendimiento del rol, existiría una dificultad real por parte de sus padres de acompañarlos en su proceso educativo, prueba de ello es la relevancia que se le asignaba a la asistencia "a reuniones de apoderados y entrevistas con el profesor", informado como lo más importante en la casi totalidad de los casos (90,2\%). Análisis que sintoniza con lo expuesto por Río (2010) en cuanto a que las familias no solo no quieren participar del proceso educativo, sino que muchas veces no pueden o no saben cómo hacerlo.

Por otra parte, la menor importancia atribuida actualmente a las reuniones de apoderados es consistente con el discurso científico social que se difunde desde la sociología, la antropología y la psicología. Se destacan aquí los trabajos de Fernández (2007), Garreta (2008), Gubbins (2012), Rivas (2007), que informan respecto de los cambios que ha experimentado la familia no solo en términos de estructura, sino también en las formas de relacionarse con el profesorado y la escuela, siendo esperable, por tanto, que no consignen este espacio como el más relevante. Más aún cuando es el mismo profesor quien asume un rol protagónico desde el saber experto, donde el vínculo familia-escuela se limitaría a encuentros informativos, formalmente normados, pero esporádicos. A ello se suma el hecho de que estos futuros docentes, en tanto que sujetos en formación, no han tenido la experiencia de relacionarse con los padres y apoderados y tampoco es una temática que se aborde de forma regular y explícita durante su formación universitaria.

En conclusión y no obstante los sesgos evaluativos que pudieran exagerar acríticamente la experiencia familiar propia respecto de la ajena en lo que a cumplimiento del rol parental educativo se refiere, lo cierto es que la función y responsabilidad de las familias en cuanto agente que acompaña y fortalece el quehacer docente, es consistente también con los discursos teóricos que se han desarrollado desde la psicopedagogía y psicología de la educación, tal como lo evidencian los trabajos de Marchesi (2000), Martín y Coll (2003), entre otros. No obstante ello, este discurso ha encontrado dificultades para permear los procesos de formación inicial docente de manera que se traduzcan en pautas de actuación concreta del quehacer en aula, operacionalizando la abstracción teórica. En su discurso pedagógico, los profesores se apropian de nuevos y actuales paradigmas teóricos, pese a ello se constata cierto tradicionalismo y visos conservadores que tienen y hacen de la relación familia-escuela.

\section{Conclusiones}

Los profesores en formación reconocen la importancia que revisten los padres en la tarea de acompañamiento y reforzamiento del quehacer educativo en cuanto, desde los objetivos trazados en la escuela, estos padres implementan estrategias y habilidades que, de modo adaptativo y flexible, obran en sintonía con las necesidades educativas de sus hijos a fin de aprovechar las oportunidades que brinda la formación escolar. Este rol parental educativo es concebido siempre como complementario a la labor docente desde fuera del espacio escolar y con una función estrictamente informativa.

Por otra parte, un rol parental consultivo o propositivo transgrede las fronteras normativas de la escuela y se constituye en una injerencia no de- 
seable que genera ruidos, incertidumbre y molestia, que terminan por alterar la relación familia-escuela expresada en un cuestionamiento permanente del quehacer del profesor. Bajo dicho nivel de participación se concibe a los padres como agentes disruptivos y de accionar amenazante.

Las percepciones atributivas al rol parental educativo por parte de los futuros profesores se vislumbran ligadas al éxito escolar del pupilo o alumno, siendo expresión del discurso académico que otorga a la familia un rol preponderante en la instalación de hábitos de estudio y desarrollo de habilidades cognitivas. Sin desconocer estos atributos, la excesiva relevancia asignada se tiende a constituir también en una suerte de externalización de responsabilidades frente al fracaso académico.
Las familias son percibidas como agentes claves, no obstante evidenciarse una imagen desmejorada respecto del cumplimiento del rol parental educativo en la actualidad, en clara discontinuidad con la experiencia propia que suele tener una apreciación evaluativa más favorable y más alejada aun respecto del estándar concebido como ideal.

Por último, la importancia atribuida al rol parental educativo señala la necesidad de que la problemática familia-escuela, así como sus bondades, dificultades y atributos, permee los procesos de formación inicial docente e irrumpa expresamente en el currículo, de manera tal que los futuros profesores se apropien de ello, tanto en lo teórico discursivo, como en la praxis pedagógica propiamente tal.

\section{Referencias}

Armas, N. (2012). Percepción del profesorado sobre la participación de las familias en la escuela, especialmente de aquellas en situación de riesgo psicosocial. IPSE-ds, 5, 9-23.

Aubert, A., Elboj, C., García, R. y García, J. (2010). Contrato de inclusión dialógica. Revista interuniversitaria de formación del profesorado, 67 (24,1), 101-111.

Bas, E. y Pérez, M. (2010). Desafíos de la familia actual ante la escuela y las tecnologías de información y comunicación. Educatio Siglo XXI, 28 (1), 41-68.

Bauman, Z. (2002). Modernidad líquida. México: Fondo de Cultura Económica.

Bellei, C., Muñoz, G., Pérez, R.y Raczinsky, D. (2003). Escuelas efectivas en sectores de pobreza. En Hevia, R. (ed.). La educación en Chile Hoy (pp. 347-373). Santiago: Universidad Diego Portales.

Bellei, C., Gubbins, V. y López, V. (2002). Participación de los Centros de Padres en la educación: expectativas, demandas, desafíos y compromisos. Santiago: CIDE / Unicef.

Bolívar, A. (2006). Familia y escuela: dos mundos llamados a trabajar en común. Revista de Educación, 339, 97-118.

Cárcamo, H. (2012). La persona educada desde la perspectiva de los futuros titulados en maestro de primaria. Tempora, 15, 107-123.

Cárcamo, H. (2013). Ciudadanía en la formación inicial docente: imágenes sobre la formación para la ciudadanía en la escuela y sus procesos de configuración [Tesis Doctoral]. Departamento de Antropología Social, Universidad Complutense de Madrid. 
ISSN 0123-1294 | Educ.Educ. Vol. 18. No. 3 | Septiembre-Diciembre de 2015 | pp. 456-470.

Universidad de La Sabana | Facultad de Educación

Cerletti, (2010). Familias y escuelas: aportes de una investigación etnográfica a la problematización de supuestos en torno a las condiciones de escolarización infantil y la categoría familia. Intersecciones en antropología, 11, 185-198.

Cornwall, A. (2002). Making spaces, changing places: Situating participation in development. IDS Working Paper, 170, 1-44.

Cox, C. (2003). Las políticas educacionales de Chile en las últimas dos décadas del siglo XX. En Cox, C. (ed). Politicas educacionales en el cambio de siglo. La reforma del sistema escolar en Chile (pp. 19 -114). Santiago: Editorial Universitaria.

Donoso, S. (2004). Reforma y política educacional en Chile. 1990-2004: el neoliberalismo en crisis. Estudios Pedagógicos, XXXI (1), 113-135.

Epstein, J. (1995). School, family, community partnerships: caring for the children we share. Phi Delta Kappa, 76 (9).

Feito, R. (2010). Familias y escuela. Las razones de un desencuentro. Revista Educación y Futuro, 22, 701-712.

Fernández, M. (2007). Educar es cosa de todos: escuela, familia y comunidad. En Garreta, J. (ed.). La relación familia-escuela (pp.13-32). Lleida: Ediciones Universidad de Lleida.

Gallardo, G. (2006). La historia de la relación familia escuela. Valoras UC.

Garreta, J. (2008). La participación de las familias en la escuela pública. Las asociaciones de madres y padres del alumnado. Madrid: CIDE-CEAPA.

Garreta, J. (2009). Escuela y familias inmigradas: relaciones complejas. Revista Complutense de Educación, 20 (2), 275-291.

Garreta, J. y Llevot, N. (2011). Inmigrants families and the schoolin Spain: Dynamics and factors that influence their relations. Journal of Educational, Cultural and Psychological Studies, 4, 47-67.

Gaventa, J. (2005). Reflections of the uses of the 'power cube' approach for analyzing the spaces, places and dynamics of civil society participation and engagement. CFP Evaluation Series (2003-2004), 4.

Gubbins, V. (2012). Familia y escuela: tensiones, reflexiones y propuestas. Docencia, 46.

Jociles, M., Franzé, A. y Poveda, D. (2012). La diversidad cultural como problema: representaciones y prácticas escolares con adolescentes de origen latinoamericano en Madrid. Revista Alteridades, 22 (43), 63-78.

Londoño, L.y Ramírez, L. (2012). Construyendo relación familia-escuela:consideraciones a partir de intervención interdisciplinaria en el Colegio Bello Oriente en Medellín, Colombia. Revista Virtual Universidad Católica del Norte, 36, 193-220.

Marchesi, A. (2000). Controversias en la educación española. Madrid: Alianza. 
Martín, E. y Coll, C. (2003). Aprender contenidos, desarrollar capacidades. Intenciones educativas y planificación de la enseñanza. Barcelona: Edebé.

Mineduc (2004). Reuniones de padres, madres y apoderados/as: un espacio de aprendizaje mutuo. Todos comprometidos con la calidad de la educación. Santiago: Mineduc, Serie Crecer en Familia.

Moscoso, M. (2009). Perspectivas de padres y madres ecuatorianos sobre el contrato pedagógico entre la institución escolar y la familia en un contexto migratorio. Revista Papeles de Trabajo sobre Cultura, Educación y Desarrollo Humano, 5 (1).

Núñez, M., Arévalo, A.y Ávalos, B. (2012). Profesionalización docente: ¿es posible un camino de convergencia para expertos y novatos? REDIE, 14 (2), 10-24.

Olmsted, P. (1991). Parent involvement in elementary education: Findings and suggestions from the follow Through Program. The Elementary School Journal, 91 (3), 221-231.

Oraisón, M. y Pérez, A. M. (2006). Escuela y participación: el dificil camino de la construcción de ciudadanía. Revista Iberoamericana de Educación, 42, 15-29.

Pascual, C. (2002). Pedagogía crítica en la formación del profesorado de educación física. Revista Interuniversitaria de Formación del Profesorado, 43.

Pascual, B.y Gomila, M. (2012). La construcción del discurso social de los futuros docentes en relación a la participación de las familias. Tempora, 15, 93-106.

Pérez, L., Cortese, I.y Gallardo, G. (2007). Manual para profesores jefe. Construyendo una alianza efectiva:familiaescuela. Santiago: Unicef.

Poveda, D., Jociles, M. y Franzé, A. (2014). Inmigrant students and the ecology of externalization in a secondary school in Spain. Anthropology and Education Quarterly, 45 (2), 185-202.

Pozo, J. (1996). Aprendices y maestros. Madrid: Alianza Editorial.

Ramírez, H. (2008). Relación familia-escuela desde la perspectiva de los padres [Tesis para optar al Grado de Magíster en Educación]. Universidad del Bío-Bío, Chile.

Río, M. (2010). No quieren, no saben, no pueden: categorizaciones sobre las familias más alejadas del campo escolar. Revista Española de Sociología, 14, 85-105.

Rivas, A. (2007). Transformaciones socioculturales y cambios familiares: continuidades y rupturas. En Lisón (ed.). Introducción a la antropología social y cultural. Teoría, método y práctica (pp. 105-128). Madrid: Akal.

Roldán, M. (2008). Familia y escuela como agentes de socialización. En López, M. (ed.). Familia, escuela y sociedad. Responsabilidades compartidas en la educación (pp. 95-137). Madrid: Ediciones Cinca. 
ISSN 0123-1294 | Educ.Educ. Vol. 18. No. 3 | Septiembre-Diciembre de 2015 | pp. 456-470.

Universidad de La Sabana | Facultad de Educación

Sampé, M., Arandia, M. y Elboj, C. (2012). Actuaciones educativas que están consiguiendo éxito educativo en centros educativos con alumnado inmigrante. Revista Interuniversitaria de Formación del Profesorado, 73 $(26,1), 119-132$.

Santín, D. (2006). Familia, escuela y fracaso escolar. En López, M. (ed.). La familia en el proceso educativo (pp. 181222). Madrid: Ediciones Cinca.

Santos Guerra, M. (2010). La formación del profesorado en las instituciones que aprenden. Revista Interuniversitaria de formación del profesorado, 68 (24, 2), 175-200.

Torres, J. (2000). Dilemas y necesidades de formación del profesorado en el marco de una escuela comprensiva que atiende a la diversidad. Revista Enseñanza, 18, 183-199.

Vera, M. (1987). La didáctica en cuestión. Investigación y enseñanza. Madrid: Narcea.

Walker, J. M. T. y Hoover-Dempsey, K. V. (2008). Parent involvement. En Good, T. (ed.). 21st Century Education: A referent handbook (pp. 382-392). Thousand Oaks: Sage Publications. 